\title{
A Multiagent System for Edge Detection and Continuity Perception on Fish Otolith Images
}

\author{
Anne Guillaud \\ École Nationale d'Ingénieurs de Brest, Technopôle Brest-Iroise, BP 30815, 29608 Brest Cedex, France \\ Email: Anne.Guillaud@ifrance.com \\ Herve Troadec \\ Institut Français de Recherche pour l'Exploitation des Mers, Center de Brest, Laboratoire de Sclérochronologie \\ des Animaux Aquatiques, BP 70, 29280 Plouzané, France
}

\begin{abstract}
Abdesslam Benzinou
École Nationale d'Ingénieurs de Brest, Technopôle Brest-Iroise, BP 30815, 29608 Brest Cedex, France

Email: benzinou@enib.fr

Jean Le Bihan

École Nationale d'Ingénieurs de Brest, Technopôle Brest-Iroise, BP 30815, 29608 Brest Cedex, France Email: jean.le_bihan@enib.fr

\author{
Vincent Rodin \\ École Nationale d'Ingénieurs de Brest, Technopôle Brest-Iroise, BP 30815, 29608 Brest Cedex, France \\ Email: rodin@enib.fr
}

Received 5 July 2001 and in revised form 26 January 2002

\begin{abstract}
We present an algorithm for fish otolith growth ring detection using a multiagent system. Up to now, the identification of growth rings, for age estimation, is routinely achieved by human readers, but this task is tedious and depends on the reader subjectivity. One of the major problems encountered during an automatic contour detection is the lack of ring continuity perception. We present an approach to improve this continuity perception based on a $2 \mathrm{D}$ reconstruction of rings using a multiagent system. The originality of the approach is to use local edge detection achieved by agents and combine it with continuity perception that active contours allow.
\end{abstract}

Keywords and phrases: edge detection, multiagent system, otolith.

\section{INTRODUCTION}

The growth of the otolith is an accretionary process. The otolith structure is made of alternative opaque and translucent concentric rings. The purpose of growth rings identification is to acquire data on age and growth of fish population. Such data are needed in a great number of biological and ecological studies and to improve stock management. Up to now, this analysis has been mainly limited to a ring count. Ring continuity is a major concept on which readers base their ring detection.

This paper presents an approach to this continuity perception based on the 2D reconstruction of rings.

In 1996 Rodin et al. [1] tried to reconstruct the rings in polar coordinates using a graph construction, by connecting nodes obtained with a primary segmentation of the image. Most of rings were detected on young individuals, but problems were encountered with older individuals, which have very thin marginal rings. In 1997, Benzinou et al. [2] applied a deformable model (Locally Deformable B-Bubble Model) to otolith images. The model was initialized at the growth center of the otolith and then inflated by computing local forces based on the gray levels and on the global shape of the otolith. Results were encouraging but sometimes drifting of points from the inflated shape could be observed. To tackle this problem, another method inspired by [3] has been proposed in [4]. The external otolith edge is used as a template which is reduced by a homothetic transform centered around 
the nucleus, which is the otolith growth starting point. As the shape of the ring is forced to be similar to the external contour, drifting of this shape points is thus avoided. Nevertheless, the rings shape can only be approximately determined using this method. Therefore, the last rings that are very thin and only distinct around the main growth axis (Figure 1) are not well detected.

Another type of methods has recently been set for detecting features in images, which are based on multiagent systems $[5,6,7,8,9]$. Multiagent systems foundations can be found in different research themes, such as distributed artificial intelligence or artificial life [10]. An agent is an entity which can be virtually or physically embodied, evolving in an environment which can contain other agents. Agents are generally autonomous, which means that they do not need external intervention to act according to the data that they perceive [11]. A multiagent system is composed of an environment, passive objects situated in this environment, at least two agents which can act on these objects, and relations between all entities of the system. These agents achieve quite simple actions, but by sharing their results, their work can bring to a more complex process. In [9] autonomous agents were used to detect homogeneous regions in brain scan images. Each agent can achieve tests on pixels around it in a circular neighborhood, as computing the variance or the mean gray level. If it finds that its neighborhood satisfies the conditions to be a region, the central pixel will be marked and new agents will be generated to grow the region. If the agent does not recognize a region, it will move to another place. This method is well adapted for brain scan images, because of regions characteristics regularity for tumors, sane parts, and so forth. In [8], a multiagent system is used to segment cytological images. One type of agent is defined that could be adapted to the research of four different features in cytological images, as the nucleus or the background. The discrimination is also mainly based on the regions characteristics. On the opposite, if we consider that otolith images can be divided in two types of regions: dark rings and light rings, it will be almost impossible to find some statistical characteristics that would always fit to each type of region. For example, as the mean gray level increases from the nucleus to the edge, the mean value of a dark ring near the edge can be superior to the mean value of a light ring near the nucleus (Figure 1). In [6], a multiagent system is proposed to detect concentric rings that can be found in natural objects such as tree trunks. Each agent can move around in its environment which is a grayscale image; its two square-shaped sensors on the pixels of the image allow it to follow light rings (light agents) or dark rings (dark agents) by moving in the direction of the lighter pixels for light agents (resp., darker pixels for dark agents). If the agents have gone over a loop, they can validate their path as a ring. The advantage of this method is to detect very quickly circular structures (a few seconds). This property is interesting for an application on otolith images featuring concentric rings. Nevertheless, on such images agents encounter problems to find all loops for old individuals, with very thin and poor contrasted rings (Figure 1). This difficulty was also observed

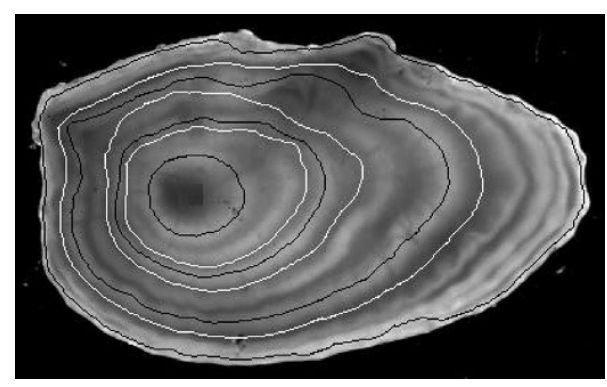

FIGURE 1: Rings detected by free agents on an eight-year-old individual.

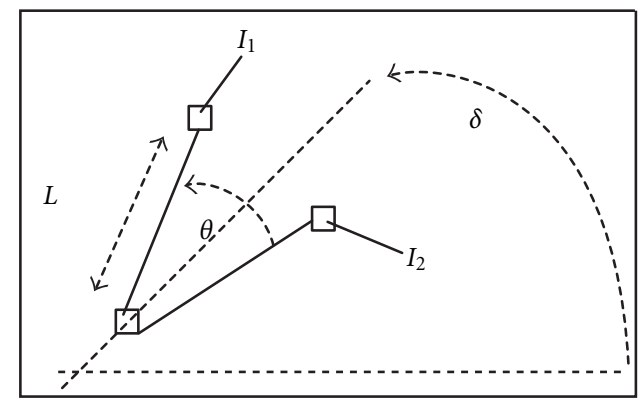

Figure 2: Agent sensors.

with other methods dedicated to otoliths image processing $[1,2,4]$.

To tackle this problem, we present in this paper an adaptation of the multiagent system to otolith growth ring detection, taking into account high level information (shape of the otolith edge and position of the growth center).

\section{DESCRIPTION OF THE INITIAL MULTIAGENT SYSTEM}

As explained above, the system is composed of reactive agents. Each agent has three sensors allowing it to get information about its environment (Figure 2). One unit sensor (one pixel) allows it to locate itself on the image. Two unit sensors are located in front of the agent and distant one from the other. The angle formed between these two sensors equals $\theta$. They return the grayscale levels on the part of the image where they are located.

A dark agent tries to move where the values returned by the sensors are minimal. A white agent does the opposite. The agents deviation is proportional to the gray level difference between the two sensors. The following equation explains how an agent computes its orientation at step $t$ depending on its orientation at step $t-1$ and on the difference of intensity between its two sensors:

$$
\delta_{\text {agent }}(t)=\delta_{\text {agent }}(t-1)+\frac{\left(I_{1}-I_{2}\right)}{P},
$$

where $P$ is a coefficient which allows to tune this deviation 
and can be adapted to the length $L$ of the sensors, $I_{1}$ (resp., $I_{2}$ ) is the intensity read by the sensor 1 (resp., 2). The tuning of parameter $P$ is explained in [12]. Depending on the data obtained with its sensors and on its internal states, each agent takes decisions. Those internal states are represented by a finite state machine. At the beginning the agent is initialized at random on the image and at the end it can validate a ring if it has gone over a loop or dies if it has not found any ring, after a certain time. The number of agents has been set to five for this particular application. A more important number of agents would not improve much results, as interactions between agents are limited. When an agent dies (if it is too old or if it has found a ring), a new agent is initialized randomly on the image.

Although this detection method is very quick, problems appear when rings are discontinuous, so that agents cannot cover the whole ring to come back to their initial position (Figure 1). Only distinct rings are easily detected. Nevertheless, agents may have detected an important part of the ring. We will call agents using this behavior free agents.

We present a new way to exploit the behavior of agents using an a priori knowledge about otolith growth.

\section{USE OF HIGH LEVEL INFORMATION}

As other calcified structures (scales, fin rays, vertebrae), the otolith is composed of concentric rings which appear each year. Therefore, the shape of the rings is quite parallel to the global shape of the otolith (Figure 1). Otolith growth starts from its center, which is called nucleus. By comparing the direction of the agent turning around this nucleus to the $\mathrm{di}$ rection of the contour of the otolith, a decision is taken to recognize whether its path is correct or not.

Some previous steps are necessary to record information concerning the shape of the otolith and the position of its nucleus, and are described below.

\subsection{Preliminary steps}

(a) The contour of the otolith is detected and its coordinates are recorded.

(b) Agents with sensors on the image pixels (Ballet et al. [6]) try to detect concentric rings and the coordinates of the smallest one are recorded.

(c) The nucleus is searched inside the smallest ring. The first white ring is usually well contrasted and easily validated by agents. Then we can search the minimum gray level point in a little neighborhood around the middle of the ring, which corresponds to the nucleus (Figure 3).

(d) Knowing the position of the nucleus and the coordinates of points constituting the contour of the otolith, the orientation of little segments composing the contour is computed all around the otolith and this information is recorded on a single image. Thus, this image is divided in sectors going from the nucleus to two close points of the contour. In every sector the local orientation of the contour is inscribed (Figure 4).

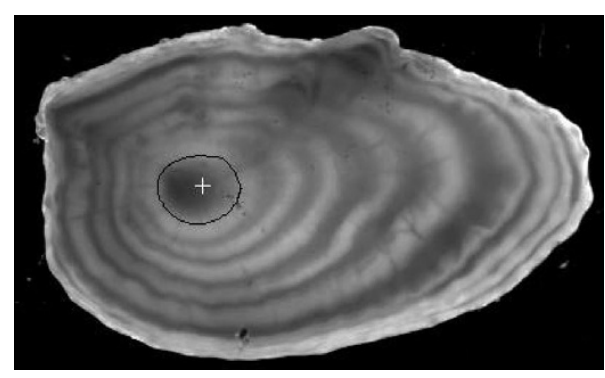

FIGURE 3: First ring and nucleus detection.

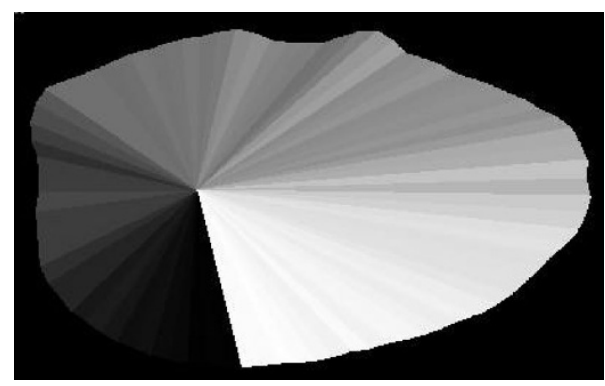

FIGURE 4: Image of local orientations.

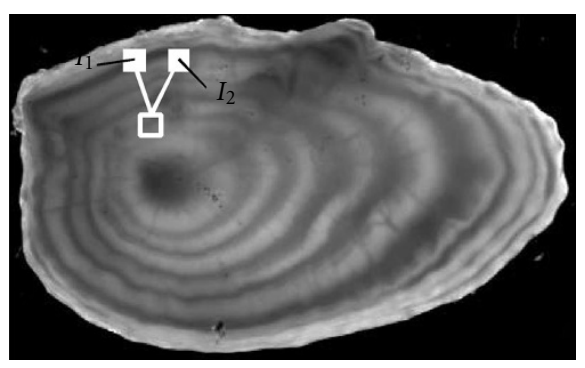

FIGURE 5: Agent sensors on the gray level image.

When this image is created, the agents will have to detect growth rings using these high level knowledge. We propose two different methods to reach this goal: Directed agents and Reconstructed path.

\subsection{Directed agents}

In this approach, agents direction is in the same time influenced by local gray levels and by the orientation of the external otolith edge. The following equation explains how an agent computes its local orientation $\delta$ at step $t$ depending on its orientation at step $t-1$, and on the gray levels of its two sensors (Figure 5):

$$
\delta_{\text {local }}(t)=\delta_{\text {agent }}(t-1)+\frac{I_{1}-I_{2}}{P} .
$$

This local orientation is compared to the orientation of the external contour in the area of the image where the agent is located (Figure 6). This angle is proportional with the gray 


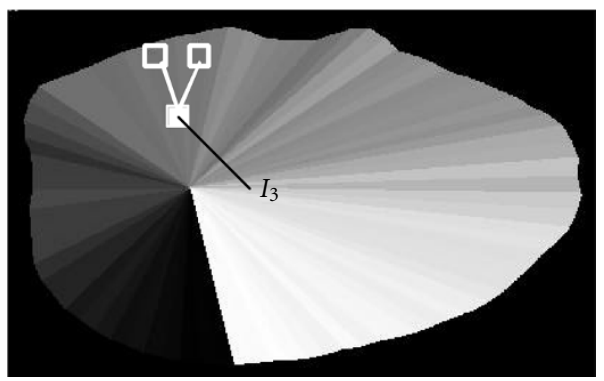

FIGURE 6: Sensor of the same agent on the orientation image.

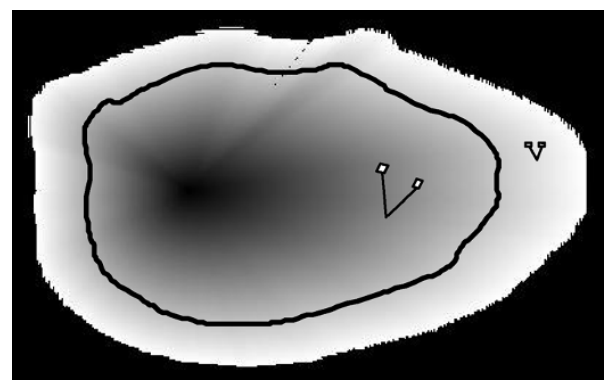

FIGURE 7: Image of the localization divided in two areas for old individuals; size of the agents sensors in each case.

level on the orientation image:

$$
\delta_{\text {contour }}=2 \pi \times \frac{I_{3}}{255} .
$$

The final agent orientation will be computed using both local and high level orientation, as explained in

$$
\delta_{\text {agent }}(t)=\delta_{\text {local }}(t)+\alpha\left(\delta_{\text {contour }}-\delta_{\text {local }}(t)\right) .
$$

In this equation $\alpha$ is a coefficient which varies from 0 near the nucleus to 1 near the external edge. This point expresses the growth of otolith from the nucleus to the edge: the shape of last rings is closer to the contour than the shape of first rings. Thus the shape constraint gets stronger when an agents move in the direction of the external edge. In Section 3.4, we explain how $\alpha$ can be computed using an image of localization (Figure 7).

Rings are validated when agents have gone over a loop and find again their initial position.

\subsection{Reconstructed path}

In this approach, agents are also aware of the external contour's orientation, but their direction depends only on the gray levels of the otolith image. Agents record their path in an image by increasing the gray level of a pixel each time they go on it (Figure 8). At the end of the processing, this image will be thresholded to keep the most frequented paths. During the processing, the agents also record their path in another image, whenever their local orientation is similar to the one of the external contour (they know this orientation

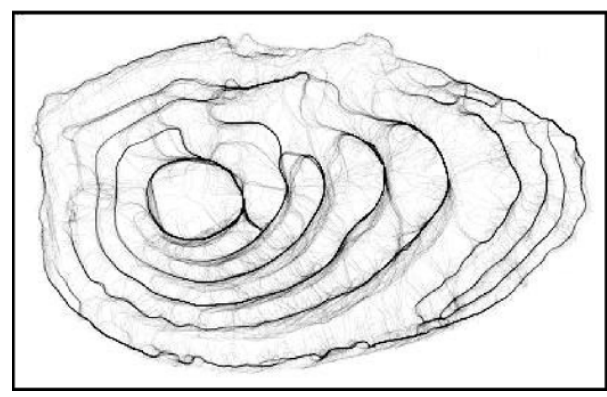

(a)

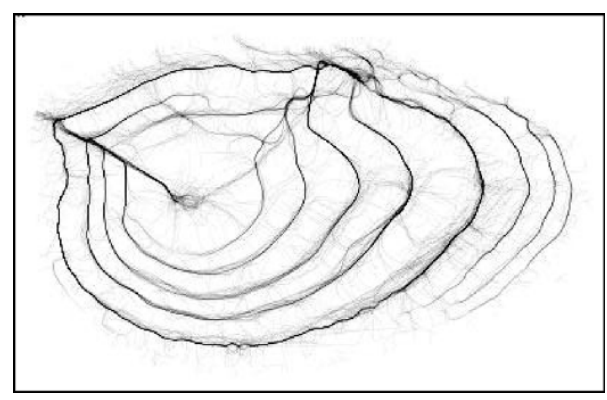

(b)

FIGURE 8: (a) Light agents path (on Figure 3), (b) dark agents paths.

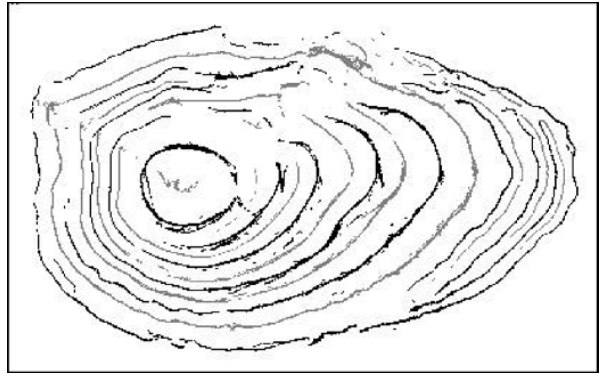

FIGURE 9: Most frequented and best oriented agents paths.

using the image created in step (d) of Section 3.1). A logical AND between this image and the thresholded path image will allow to obtain a map of growth rings edges, which correspond to the most frequented and best oriented agents paths (Figure 9). This map will be used to begin rings reconstruction, starting from the smallest one around the nucleus, and using a method similar to active contours. The shape of the current ring is inflated, by translating each point of the shape using a vector, whose direction is similar to the one of a line going through the nucleus and this point (Figure 10). Growth stops when the correspondence between the inflated shape and the pixels of the edge map is maximal. This correspondence is computed by counting the number of common nonzero pixels between the inflated shape and the edge map (Figure 9).

By counting the number of rings detected with the first or the second method, the age of the fish can be estimated.

Figure 11 illustrates the result of the method on an eightyear-old individual and on a ten-year-old individual. 


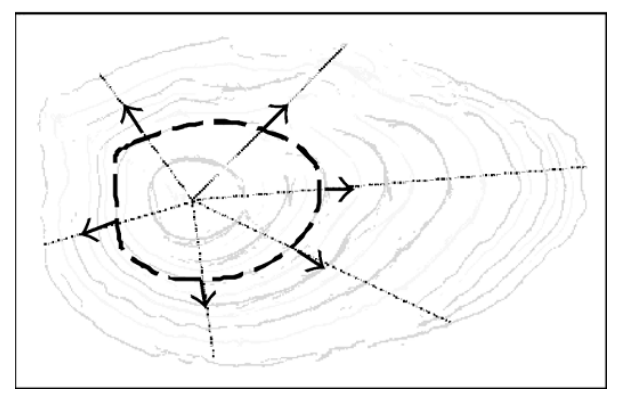

FIgURE 10: Inflation of the current ring for next ring detection.

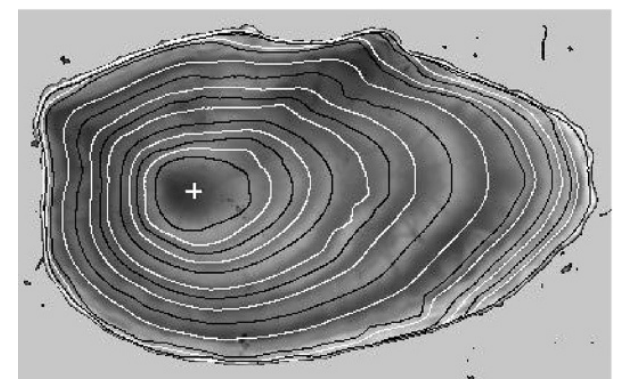

(a) Eight-year-old individual.

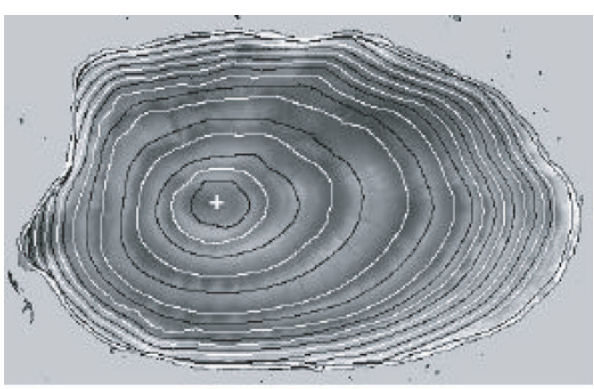

(b) Ten-year-old individual.

FIGURE 11: Rings detected with reconstructed path method.

\subsection{Agents parameters adjustment}

The width of rings appearing during the fish growth decreases when the fish gets older. Therefore, the size of agents sensors needs to be adapted in order to detect large rings near the nucleus and thin rings near the edge. Rings width can be approximated according to their position in the otolith using a growth model, but variability is very important between individuals. Nevertheless, the size of the otolith allows to estimate roughly the number and the width of rings. Knowing the position of the nucleus and the coordinates of the otolith edge, it is possible to create an image which will inform the agent about its relative position on the otolith (Figure 7). In this image the gray level increases from 0 on the nucleus to 255 at the otolith edge. The agents will be provided with one sensor on this image. As the gray level of this image increases from the nucleus to the edge, the intensity read by the sensor will allow the agent to locate itself in the image. As it was

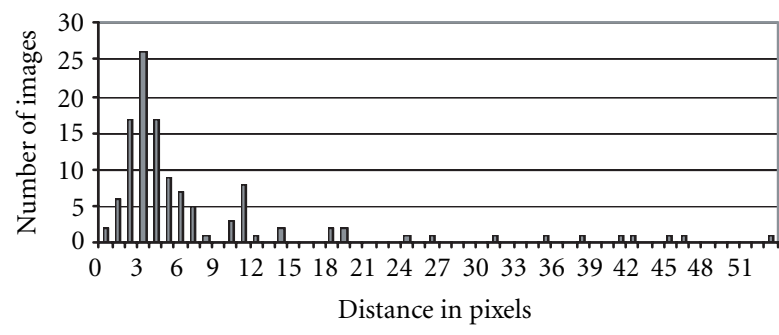

Figure 12: Distribution of the distance between the nucleus detected automatically and the nucleus detected by a human reader.

shown in $[12,13]$, agents can detect edges of different widths with the same parameters. Thus when otoliths are young, which means that rings width is quite constant, agents can detect all rings with only one set of parameters, but for older individuals they have to reduce the length of their sensors to improve thin rings detection, which appear at the end of the fish life. When the size of the main growth axis corresponds to an old individual, the image of the localization (Figure 7) will be divided in two sectors. The contour of the central area is defined by the points of the external shape reduced by an homothety with a factor of $3 / 4$. These points are also defined by the pixels having the same gray level $G_{\max }$ in this image. According to the gray level $G$ an agent reads in this image, the length $L$ of its sensors will be determined as if $G<G_{\max }$ then $L=L_{\max }$ else $L=L_{\min }$.

For our tests with plaice otolith images, the optimal values for $L_{\max }$ and $L_{\min }$ were, respectively, 8 and 2 pixels.

The localization image also allows to compute the coefficient $\alpha$ described in Section 3.2, as $\alpha=G / 255$.

\section{RESULTS AND DISCUSSION}

\subsection{Automatic nucleus detection}

The nucleus detection method has been tested by comparing the position of the nucleus automatically detected to the one of the nucleus detected by a human expert on a sample of 119 plaice otolith images. As the real position of the nucleus is unknown, we can only measure the distance between these two estimations to evaluate the method. We obtain a quite good detection of the nucleus with a 7.86 pixels mean distance between automatic and manual detection, with a $512 \times 512$ resolution (Figure 12). The images for which the detection error is quite important are very bad contrasted and the first ring is very difficult to distinguish.

\subsection{Age estimation methods}

The two methods have been evaluated by comparing the age automatically estimated on a sample of 119 plaice otolith images, from age group 1 to 13 , with the age estimated on these images by a human reader. The number of a group corresponds to the age of the fishes in the group. The image on Figure 1 represents the rings detected on an eight-year-old plaice otolith by free agents, which means that the agents have no high level information about the image they are 


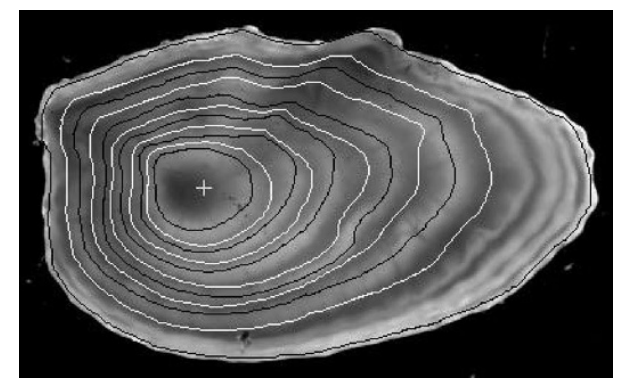

FIgURE 13: Rings detected by directed agents.

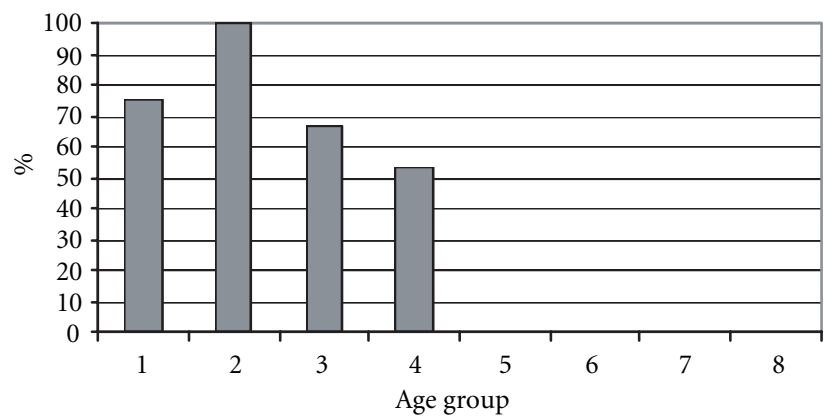

Figure 14: Percentage of good age estimation for directed agents on otoliths from age group 1 to 8 .

processing. They only try to validate loops by searching for local intensity extremes. Therefore the bad contrasted or indistinct rings are not detected.

As agents are aware of the shape of the otolith, in the directed agents method, they can go forward in areas where contrast between light and dark rings is very bad. The number of detected rings has been improved with this method and fanciful loops, that can be detected by free agents, have been avoided (Figure 13). This method is nevertheless causing a frequent under-estimation of the age of old fishes, because the condition required to validate a ring-when an agent finds again its initial position-is not easily satisfied when rings are distinct only in a restricted area of the image (Figures 13 and 14).

The reconstructed path method allows to improve the detection of these last rings, using the local detection of edges achieved by agents, and guiding the reconstruction of a ring using the shape of the previous one. The percentage of good age estimation is thus better for old individuals (Figures 11 and 15).

The methods proposed in this paper give better results than those obtained with a mono-dimensional method applied on otolith images, which is described in [14]. This method consists in searching intensity extremes on an image profile starting from the nucleus to the otolith edge. Therefore the structures continuity is not taken into account.

The reconstructed path method also presents better results than those presented in [4] (deformable templates), mainly for old individuals because the shape of rings is more correctly detected. Otherwise, the graph method [1] gives

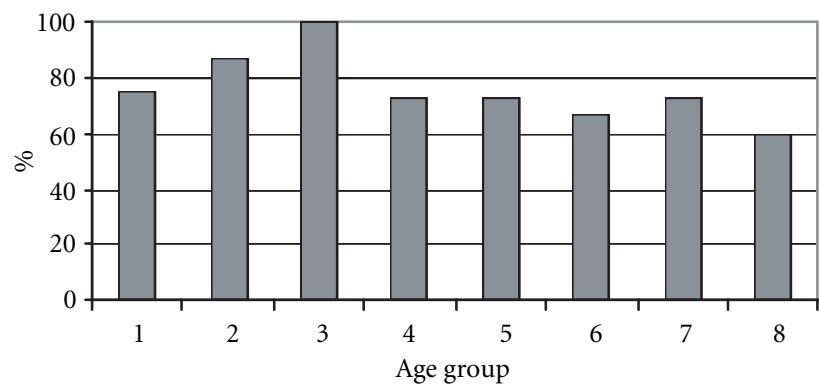

FIGURE 15: Percentage of good age estimation for the reconstructed path on the same images.

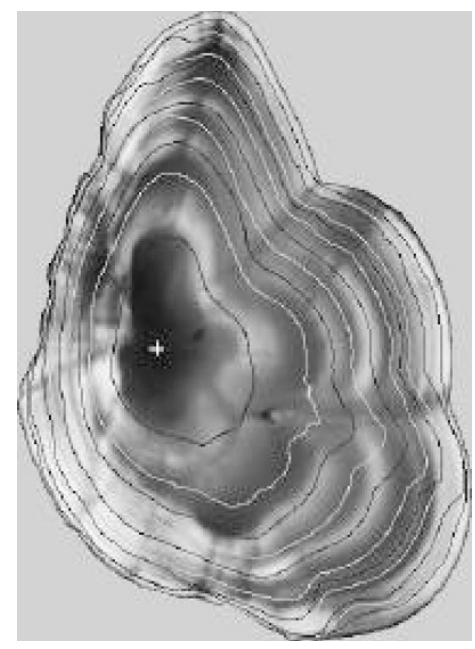

FIGURE 16: Result of the reconstructed path method on a pout fish otolith image.

similar results with those of the reconstructed path, but the latest is less time-consuming ( 2 minutes maximum against 12 minutes maximum). Moreover the graph method, which requires a polar transformation of the image using the nucleus as the center, cannot be applied to otoliths having several nuclei. Figure 16 illustrates the fact that the reconstructed path method can be used for processing more complex images such as pout fish otolith images. In this image, the otolith has actually three nuclei, but agents are still able to detect the first white ring. Then the center of this ring is searched; even if it is not meaningful, it is helpful to start ring reconstruction.

\subsection{Discussion}

In Table 1 the percentage of good age estimation obtained with the two methods presented in this paper is compared with methods previously developed. The $1 \mathrm{D}$ method is a mono-dimensional method based on the extraction of radials starting from the nucleus and going to the external edge to search extremes in these radials [14]. This method cannot take into account structures continuity and the results are lower than those obtained with the methods we propose.

The template method uses the shape of the external 
TABle 1: Performance of different age estimation methods.

\begin{tabular}{|c|c|c|c|c|c|}
\hline$N=110$ & 1D method & Template method & Graph method & Directed Agents & Reconstructed Path \\
\hline \multirow{2}{*}{1 to $3 y$} & $R=50$ & $R=100$ & $R=90$ & $R=81$ & $R=87$ \\
\hline & $\mathrm{AM}=[-2,+1]$ & $\mathrm{AM}=[0,0]$ & $\mathrm{AM}=[-1,+1]$ & $\mathrm{AM}=[-1,+1]$ & $\mathrm{AM}=[0,+1]$ \\
\hline \multirow{2}{*}{1 to $5 y$} & $R=50$ & $R=80$ & $R=80$ & $R=59$ & $R=82$ \\
\hline & $\mathrm{AM}=[-2,+2]$ & $\mathrm{AM}=[-2,0]$ & $\mathrm{AM}=[-1,+1]$ & $\mathrm{AM}=[-3,+1]$ & $\mathrm{AM}=[-1,+2]$ \\
\hline \multirow{2}{*}{5 to $8 y$} & & $R=20$ & $R=60$ & $R=0$ & $R=68$ \\
\hline & & $\mathrm{AM}=[-5,+1]$ & $\mathrm{AM}=[-2,+2]$ & $\mathrm{AM}=[-7,-1]$ & $\mathrm{AM}=[-1,+2]$ \\
\hline Computing time $t$ & $t=7,4 \mathrm{~s}$ & $2 \min <t<4 \min$ & $t<12<\min$ & $7 \mathrm{~s}<t<15 \mathrm{~s}$ & $30 \mathrm{~s}<t<2 \mathrm{~min}$ \\
\hline
\end{tabular}

$R$ : percentage of good age estimation

AM: maximal error amplitude

otolith edge reduced by an homothetic transform centered on the nucleus, in order to search the position of growth rings [4]. Their position is determined by minimizing an energy function which is computed with the gray levels of the image. This method can only give an approximate shape to rings and presents quite low results for age groups from 5 to 8 , in comparison with the reconstructed path method.

The graph method uses a polar coordinates transform to extract radials starting from the nucleus and going to the edge in the original image to display them one under the other in the final image [1]. Peaks, corresponding to light rings and valleys corresponding to dark rings are then extracted using morphological transforms. Objects are then labelled and closest objects are connected to reconstruct rings. This method gives results similar to the reconstructed path method, but cannot be applied to otoliths having several nuclei because of the polar coordinates transform (Figure 16).

The deformable model method presented in [2], inspired by [15], cannot be easily compared with the methods we propose because it has not been evaluated on a sample of test images.

However, this method may encounter problems to detect thin rings which are visible around the main growth axis for old individuals, as the energy function computed to inflate the model uses gray levels all around the otolith.

Processing time for estimating age with directed agents and reconstructed path on otolith images may be longer than the time necessary for a human person to estimate age. Nevertheless, a human person is not able to draw precisely by hand the position of the rings, or this would be a very long and tedious task. Moreover, the same person, at different times, or two different people, can make different estimations for the same image.

Ring detection on otolith images is a very complex problem, as the number and the shape of structures to detect in an image is a priori unknown. Thus it is essential to use high level knowledge to improve this detection. For this reason it may be difficult to compare the methods we propose in this paper with more general methods presented in the literature. However, agents present the advantage to perceive locally roof edges and structures continuity at the same time. Agents can also cooperate by sharing their work on the same image. The use of snake curves thrown on the image instead of agents could be suggested. Nevertheless, snakes detect step edges, and in our application detection of peaks and valleys is more appropriate to localize growth rings. Moreover if agents are initialized on the image in an area where no ring is visible, they are able to move from this area, which would be more difficult for a snake, if its energy function does not allow it to evolve.

\section{CONCLUSION}

We have developed a method to perceive continuity of contours in textured, noisy, and low contrast images. Previous methods needed operator intervention to give the nucleus position whereas the pointing is automated here. The agents are able to detect local edges in the image, while perceiving their continuity by the way they move. This property is very interesting for processing otolith images, which are noisy and textured. Because they are too sensible to noise and texture, classical edge detection operators cannot be used with such images. Agents can also adapt locally the size of their sensors according to the ring width.

In the future we intend to find a new criterion for the agents to validate a ring, which should be more robust than the fact that the agents have to find again their initial position.

Plaice otolith images were used to develop automatic age estimation methods as they are quite simple compared to other species. Nevertheless, we hope to be able to adapt the method to more complex species (Figure 16), which can have several nuclei and less regular shapes.

\section{REFERENCES}

[1] V. Rodin, H. Troadec, H. de Pontual, A. Benzinou, J. Tisseau, and J. Le Bihan, "Growth ring detection on fish otoliths by a graph construction," in Proc. IEEE International Conference on Image Processing, vol. II, pp. 685-688, Lausanne, Switzerland, September 1996.

[2] A. Benzinou, H. Troadec, J. Le Bihan, V. Rodin, H. de Pontual, and J. Tisseau, "The locally deformable B-bubble model: An application to growth ring detection on fish otoliths," in Proc. 10th Scandinavian Conference on Image Analysis, vol. I, pp. 181-187, Lappeenranta, Finland, June 1997. 
[3] A. L. Yuille and P. W. Hallinan, "Deformable templates," in Active Vision, A. Blake and A. L. Yuille, Eds., Artificial Intelligence Series, chapter 2, pp. 21-38, MIT Press, Cambridge, Mass, USA, 1992.

[4] H. Troadec, A. Benzinou, V. Rodin, and J. Le Bihan, "Use of deformable templates for otolith $2 \mathrm{D}$ growth ring detection by digital image processing: application to plaice (Pleuronectes platessa) otoliths," Fisheries Research Journal, vol. 46, no. 1-3, pp. 155-163, 2000.

[5] P. Ballet, V. Rodin, and J. Tisseau, "Edge detection using a multiagent system," in Proc. 10th Scandinavian Conference on Image Analysis, vol. II, pp. 621-626, Lappeenranta, Finland, June 1997.

[6] P. Ballet, V. Rodin, and J. Tisseau, "A multiagent system for detecting concentric strias," in Applications of Digital Image Processing XX, vol. 3164 of Proceedings of SPIE, pp. 659-666, San Diego, Calif, USA, 30 July-1 August 1997.

[7] O. Boissier and Y. Demazeau, "ASIC: An architecture for social and individual control and its application to computer vision," in Proc. 6th European Workshop on Modelling Autonomous Agents in a Multi-Agent World, pp. 107-108, Odense, Denmark, August 1994.

[8] A. Boucher and C. Garbay, "A multi-agent system to segment living cells," in Proc. 13th IAPR International Conference on Pattern Recognition, vol. III, pp. 558-562, IEE Computer Society Press, Vienna, Austria, August 1996.

[9] J. Liu, "Reactive agents for adaptative image analysis," in Proc. 2nd International Conference on Autonomous Agents, pp. 441448, Minneapolis, Minn, USA, May 1998.

[10] J. Ferber, Les systèmes multi-agents: vers une intelligence collective, InterEditions, Paris, France, 1995.

[11] P. Maes, "Modeling adaptative autonomous agents," Artificial Life Journal, vol. 1, pp. 135-162, 1994.

[12] A. Guillaud, A. Benzinou, H. Troadec, V. Rodin, and J. Le Bihan, "Parameterization of a multi-agent system for roof edge detection: An application to growth ring detection on fish otoliths," in SPIE Electronic Imaging: Science and Technology, San Jose, Calif, USA, January 2000.

[13] A. Guillaud, Un système multi-agents pour la reconnaissance de formes sur des pièces calcifiées, Aide à l'estimation de l'âge de poissons et de céphalopodes, Thèse de Doctorat, Université de Bretagne Occidentale, Brest, France, December 2000.

[14] H. C. Welleman and F. Storbeck, "Automatic ageing of plaice otoliths by means of image analysis," in Recent Developments in Fish Otoliths Research, D. H. Secor, J. M. Dean, and S. E. Campana, Eds., pp. 271-282, 1995.

[15] L. D. Cohen, "On active contour models and balloons," Computer Vision, Graphics and Image Processing: Image Understanding, vol. 53, no. 2, pp. 211-218, 1991.

Anne Guillaud was born in 1974. She has studied and taught at the École Nationale d'Ingénieurs de Brest (France). She received in 2000 the Ph.D. degree in signal and image processing.
Herve Troadec was a researcher at the Ifremer institute (France). He was specialized in schlerochronology, which is a technique consisting in reading age of fishes for fisheries assessment. He contributed to the development of a software used for processing numerical images of calcified structures. This software is used in several laboratories in Europe and Africa. His last mission consisted in an expertizing mission in Morocco for the COPEMED project of the FAO (Food and Agriculture Organization), in august 2001. He died at 41 years old in an air crash in Malaga (Spain) on the 29th of August 2001.

Abdesslam Benzinou was born, on December 7, 1970, in Casablanca, Morocco. $\mathrm{He}$ received the Ingénieur degree in electronics and software engineering from the École Nationale d'Ingénieurs de Brest and the Diplôme d'Études Approfondies (DEA) in electronics and optronics, with honours, from the Université de Bretagne Occidentale, both in 1995 in Brest, France. He received the Ph.D. degree in January 2000,

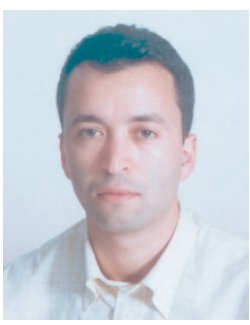
from the same university, for his work dealing with the problem of designing and realizing of a computer vision system for the recognition of patterns on calcified structures (otoliths and statoliths). In September 2000, he was engaged as a Researcher in signal processing for metrology and communication systems at SchlumbergerRMS, Chasseneuil, France. In September 2001, he joined the RESO Laboratory at the École Nationale d'Ingénieurs de Brest, Brest, France, as a Maître de Conférences. Apart from teaching, his main fields of interest include signal analysis, computer vision, pattern recognition, and related aspects of mathematical modelling and optimization.

Jean Le Bihan was born in France. He studied at the École Normale Supérieure, Cachan, and at the Universities of Orsay and Paris, and received the Agrégation of applied physics. Then he became an Assistant Professor of electronic engineering at Brest University and received the degree of Docteur d'État ès Sciences Physiques. From 1986 to 1988 , he worked as a Visiting Researcher, first at Thomson, Orsay, then at CNET, Lannion. Since 1988, as a Professor at the École Nationale d'Ingénieurs de Brest, he has been at the head of RESO Laboratory, working mainly in the area of optical communication systems and image processing.

Vincent Rodin was born on February 28 1966. Lecturer at École Nationale d'Ingénieurs de Brest (France), he is working on image processing, virtual reality, and computer simulation of biologic processes.

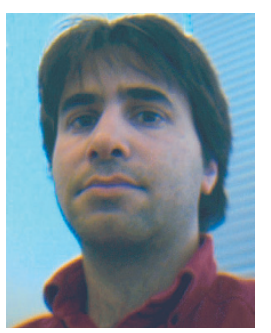

